\title{
Urinary tract infections: to prophylaxis or not to prophylaxis?
}

\author{
Giovanni Montini • Ian Hewitt
}

Received: 10 April 2009/Revised: 15 April 2009/Accepted: 17 April 2009/Published online: 15 May 2009

(C) IPNA 2009

\begin{abstract}
Urinary tract infections, with or without reflux, are the most frequent reason for long-term antibacterial prophylaxis in infants and children. When one is considering the use of prophylaxis in these children, what is clear from existing published data? Up to 2006 there were a small number of poor quality studies, with no reliable evidence of the effectiveness of antibiotics in preventing recurrent symptomatic urinary tract infections (UTIs). Since then, four randomised controlled studies, to assess the benefits, or lack thereof, of antibiotic prophylaxis, have been published. None has demonstrated a clear benefit. The following conclusions can be drawn: antibiotic prophylaxis is not indicated for children following a first febrile UTI if no or mild grade I or II reflux is present; for higher grade reflux there is no definite conclusion.
\end{abstract}

Keywords Urinary tract infection - Antibiotic prophylaxis . Bacterial resistance $\cdot$ Vesico-ureteric reflux $\cdot$ Children

In a recent poll [1], more than 11,300 readers of the British Medical Journal chose the discovery of antibiotics as the

\author{
G. Montini \\ Departments of Pediatrics, \\ Azienda Ospedaliera di Bologna, \\ Bologna, Italy \\ I. Hewitt \\ Princess Margaret Hospital, \\ Perth, Australia \\ G. Montini ( $\bowtie)$ \\ Nephrology, Dialysis Unit, Department of Pediatrics, \\ Azienda Ospedaliera Universitaria \\ Sant'Orsola-Malpighi Bologna, \\ Via Massarenti 11, Padiglione 13, \\ Bologna, Italy \\ e-mail: giovanni.montini@aosp.bo.it
}

second most important medical milestone since 1840 (15\% of the votes), while sanitation (introduction of clean water and sewage disposal) topped the poll (15.8\% of votes).

In contrast, a recent New England Journal of Medicine editorial, discussing the emergence of antibiotic-resistant organisms [2], stated that, for patients infected with multidrug-resistant bacteria, we have arrived at a point as frightening as the pre-antibiotic era. One of the most difficult situations described was the development of nosocomial gram-negative infections secondary to multidrug-resistant organisms, including Pseudomonas aeruginosa, Klebsiella spp., and Escherichia coli. In the same article the authors described adult outpatients with urinary tract infections (UTIs) due to Escherichia coli, resistant to most of the major antibiotics utilised.

In Europe a correlation between antibiotic resistance and outpatient antibiotic use has been shown, both in adults [3] and children [4]; a further study [5] suggested that children were the main consumers of antibiotics, with usage rates three-times higher than those of older patients, thereby increasing their risk of the development of resistant organisms. A role in this public health problem is played also by us paediatricians, paediatric nephrologists and urologists, who widely prescribe antibiotic prophylaxis in children following a febrile UTI [6]. In recent years it has been an everyday clinical experience to note an increasing proportion of E. coli resistant to amoxicillin and cotrimoxazole (of the 149 UTIs diagnosed in the Pediatric Emergency Unit in Padova in 2007, 49\% and 17\% were resistant to amoxicillin and cotrimoxazole, respectively) to the point that amoxicillin is no longer considered in the treatment of a first UTI.

This major public health problem of emerging bacterial resistance should be kept in mind when, following a UTI, we prescribe long-term antibiotic prophylaxis, aimed at 
preventing recurrence. We should be certain of the positive effects of this prescription, with the benefits clearly outweighing any risks.

Two recent editorials in this journal have outlined suggestions that might [7] or might not [8] decrease the occurrence of renal scarring and possible end-stage renal disease (ESRD), by identifying and treating children with vesico-ureteric reflux (VUR) and preventing recurrent UTI. It appears, therefore, that the overall importance of VUR and of UTIs is confounded. We have recently conducted a systematic review of the literature (A. Toffolo and G. Montini, data not published) on the long-term prognosis in children aged $0-16$ years with a single or recurrent episodes of febrile urinary tract infection. When we considered renal function as the outcome measure, a total of 1,662 children from 15 cohorts was identified, with a follow-up period ranging from 2-27 years. VUR was present in $90 \%$ of these children, and major scars in $40 \%$, with the incidence of chronic renal failure (CRF) ranging from $0-56 \%$ in the cohorts examined. When the outcome measure was hypertension, the incidence varied from $0-35 \%$ in 14 cohorts of 1,541 children with the same follow-up period, $77 \%$ of whom had renal scars. From this literature review it appears that conclusions cannot be drawn on the long-term consequences of UTI in children, as there was marked heterogeneity in the populations studied, diagnostic criteria employed and the evaluation of outcomes. The cohorts of children studied were often derived from tertiary institutions and specialist clinics such that they were more likely to have severe disease with high grade reflux and renal dysplasia, rather than the uncomplicated UTIs more typically seen in general practice, particularly in countries where a normal prenatal ultrasound (US) has largely excluded high-grade reflux or dysplastic kidneys. We concluded that the relationship between UTI and longterm morbidities is currently ambiguous and yet to be reliably determined. This needs to be taken into account when one is prescribing antibiotic prophylaxis in anticipation that it might prevent long-term adverse consequences, until additional trial data on the benefit-risk trade-off become available.

The role of antibiotic prophylaxis following a febrile UTI, in otherwise healthy children, implies other medical and ethical issues, particularly an aggressive diagnostic protocol [micturating cystogram and renal di-mercaptosuccinic acid (DMSA) scans] to evaluate the presence of reflux and renal damage.

When one is considering the use of prophylaxis, what is clear from existing published data? Searching the literature up to 2006, an extensive editorial commentary in this journal [9] reported that, while the strategies of antibacterial prophylaxis for the prevention of recurrent urinary tract infection were no longer universally accepted, recurrent urinary tract infections, with or without reflux, remained the most frequent reason for long-term antibacterial prophylaxis in infants and children. A recent Cochrane review [10] concluded that the small number of poor quality studies gave no reliable evidence of the effectiveness of antibiotics in preventing recurrent symptomatic UTIs. The implication of this review for future research was that there is a need for large, properly designed and randomised, parallel group, placebo-controlled, double-blind studies. In the same year a controlled, randomised trial by Garin et al. [11] showed that the use of antibiotic prophylaxis in reducing the frequency of UTIs among patients with acute pyelonephritis was not only ineffective, but also harmful, with a significant $(P=$ $0.029)$ increase in the incidence of recurrent pyelonephritis in the no-prophylaxis group. In this study the effectiveness of antibiotic prophylaxis after acute pyelonephritis was evaluated in 236 patients aged 3 months to 18 years. Furthermore, antibiotic prophylaxis was not shown to be effective in reducing the subsequent development of renal scars.

In 2007 the National Institute of Health and Clinical Excellence (NICE) guidelines for the investigation and management of childhood UTIs were published [12]. They recommended the cessation of routine antibiotic prophylaxis for paediatric UTIs.

During the past year there has been a resurgence of interest in this topic, with three prospective studies published that leave us better positioned in 2009, to assess the benefits, or lack thereof, of antibiotic prophylaxis.

Roussey-Kesler et al. [13] published a multicentre prospective, randomised, study of trimethoprim-sulphamethoxazole antibiotic prophylaxis, or no treatment, for the prevention of recurrent UTI, in 225 children aged 1 month to 3 years with grades I to III VUR, followed for 18 months. The results failed to show an overall difference in the occurrence of UTIs between the groups; however, subgroup analysis did demonstrate a significant $(P=0.013)$ reduction in UTIs in boys, which was most apparent in grade III reflux.

Pennesi et al. [14], in a multicentre, open-label, randomised, controlled trial, found no difference in the risk of having at least one pyelonephritic episode between the intervention (sulfamethoxazole/trimethoprim) and control (no treatment) groups in 100 children during a follow-up period of 4 years. The patients were younger than 30 months and had grades II, III or IV VUR, diagnosed after a first episode of acute pyelonephritis. At the end of the follow-up time, the presence of renal scars was the same in children with and without antibiotic prophylaxis.

We [15] conducted a multicentre, randomised, controlled, non-inferiority trial of antibiotic prophylaxis following a first febrile UTI. A total of 338 children aged 2 months to $<7$ years were enrolled, 309 with confirmed 
pyelonephritis on a DMSA scan; 210 patients had no reflux, and 128 patients had reflux grades I to III. There were no significant differences in the recurrence rate of UTI, or that of scarring produced by recurrent UTIs, after 12 months of follow-up. Interestingly, the risk of renal damage from repeat infections was very low (1-2\% of new scars, not at the site of the original pyelonephritis) and, per se, does not justify the need for prophylaxis in this population. In this study, as in the study by RousseyKesler et al. [13], children with grade III reflux and no prophylaxis experienced an increased number of recurrent infections, although not statistically significant, possibly because of insufficient numbers. Another point worth remembering is that antibiotic prophylaxis was associated with a higher number of febrile UTIs due to resistant organisms. All but one of the nine recurrences attributed to resistant bacteria occurred in the prophylaxis group; the one recurrence in the no-prophylaxis group was in a child who had been switched to prophylaxis.

Table 1 summarises the four prospective, randomised, controlled trials of antibiotic prophylaxis for preventing febrile urinary tract infections and pyelonephritis published to date. It is immediately apparent that, for such a common condition, few children have actually been studied in a rigorous manner, with a virtual absence of studies incorporating more severe (grades IV-V) VUR. None of the studies demonstrated a clear benefit from the use of antibiotic prophylaxis in their patient populations.

We performed a meta-analysis limited to the randomly allocated children with grades II and III reflux from these four recent trials (Figs. 1 and 2). Both analyses show that the recurrence of symptomatic UTI was the same in the antibiotic prophylaxis group as in the placebo group [risk ratio (RR) $0.90,95 \%$ confidence interval $(95 \% \mathrm{CI}) 0.42$ to
1.90 for grade II reflux and RR $0.92,95 \%$ CI 0.58 to 1.45 for grade III reflux].

There are three further randomised controlled studies on the use of antibiotic prophylaxis to prevent febrile UTIs yet to be published. The Swedish reflux study [16] was completed in December 2008, with results not yet available. This trial was developed to evaluate three different alternatives (endoscopic treatment with Deflux, antibiotic prophylaxis, and observation with treatment of each UTI) for VUR grades III or IV, in children aged 12 years. The study endpoints are rate of febrile UTIs during the follow-up period (24 months) and renal damage on DMSA scan and reflux status on voiding cystourethrography (VCUG) at the end of follow-up. Two hundred and three children have been randomly selected. This is a unique study that includes a third arm of endoscopic treatment, and it will be of much interest. We will also have data on higher grade reflux.

A second study, which has terminated the recruitment of patients, is the Prevention of Recurrent Urinary Tract Infection in Children with Vesicoureteric Reflux and Normal Renal Tracts (PRIVENT) study [17], a multicentre, double-blind, randomised, controlled trial designed to determine whether long-term use of antibiotics prevents urinary tract infection in children. Antibiotics are given daily to the children over 1 year. Recruitment for PRIVENT has now ceased, and 576 children have been enrolled in the study.

The most recent study to be initiated is a North American initiative: the Randomized Intervention for Children With Vesicoureteral Reflux (RIVUR) study [18]. It is a double-blind, randomised, placebo-controlled trial, which plans to enroll 600 children, 2-72 months of age, with grades I to IV vesicoureteral reflux after the
Table 1 Summary of the four prospective, randomised, controlled trials of antibiotic prophylaxis for preventing febrile urinary tract infections and pyelonephritis published to date (tot total number of randomly chosen patients in each reflux group, + number of children with recurrence of febrile UTIs)

\begin{tabular}{|c|c|c|c|c|c|c|c|c|c|c|c|c|c|c|c|c|}
\hline \multirow[t]{4}{*}{ Parameter } & \multirow{2}{*}{\multicolumn{4}{|c|}{$\frac{\text { Pennesi et al. [14] }}{\text { Antibiotic prophylaxis }}$}} & \multirow{2}{*}{\multicolumn{4}{|c|}{$\begin{array}{l}\text { Montini et al. [15] } \\
\text { Antibiotic prophylaxis }\end{array}$}} & \multirow{2}{*}{\multicolumn{4}{|c|}{$\begin{array}{l}\text { Roussey-Kesler et al. [13] } \\
\text { Antibiotic prophylaxis }\end{array}$}} & \multirow{2}{*}{\multicolumn{4}{|c|}{$\begin{array}{l}\text { Garin et al. [11] } \\
\text { Antibiotic prophylaxis }\end{array}$}} \\
\hline & & & & & & & & & & & & & & & & \\
\hline & \multicolumn{2}{|l|}{ Yes } & \multicolumn{2}{|l|}{ No } & \multicolumn{2}{|l|}{ Yes } & \multicolumn{2}{|l|}{ No } & \multicolumn{2}{|l|}{ Yes } & \multicolumn{2}{|l|}{ No } & \multicolumn{2}{|l|}{ Yes } & \multicolumn{2}{|l|}{ No } \\
\hline & tot & + & tot & + & tot & + & tot & + & tot & + & tot & + & tot & + & tot & + \\
\hline No VUR & & & & & 129 & 5 & 81 & 3 & & & & & 45 & 2 & 60 & 2 \\
\hline VUR grade I & & & & & 19 & 1 & 11 & 1 & 8 & 0 & 14 & 0 & 9 & 0 & 10 & 0 \\
\hline Grade II & 11 & 1 & 10 & 0 & 37 & 3 & 21 & 2 & 70 & 8 & 77 & 8 & 28 & 1 & 29 & 1 \\
\hline Grade III & 22 & 9 & 24 & 7 & 26 & 6 & 14 & 6 & 24 & 6 & 30 & 11 & 18 & 6 & 19 & 0 \\
\hline Grade IV & 17 & 8 & 16 & 8 & & & & & & & & & & & & \\
\hline All patients & 50 & 18 & 50 & 15 & 211 & 15 & 127 & 12 & 102 & 14 & 122 & 19 & 100 & 9 & 118 & 3 \\
\hline
\end{tabular}




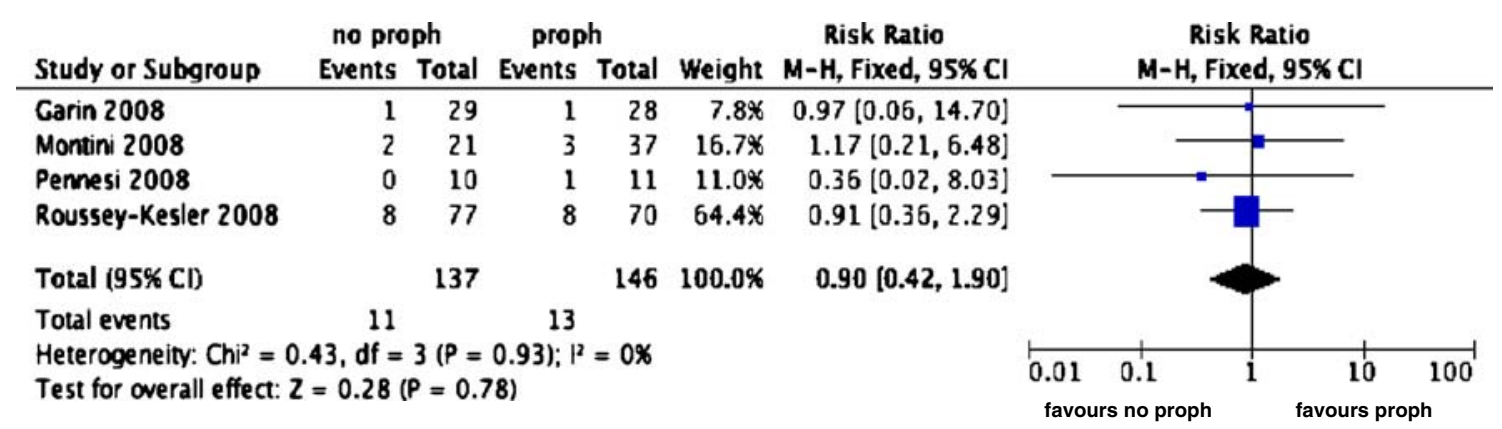

Fig. 1 Meta-analysis of recurrence of febrile UTIs during 1 year of follow-up in children with grade II reflux (proph prophylaxis)

index febrile or symptomatic UTI. The children will be randomly selected to receive either cotrimoxazole or placebo, with a follow-up period of 2 years. The primary outcome is recurrence of UTI (febrile or symptomatic), while secondary outcomes include time to recurrent UTI, renal scarring (DMSA scan), treatment failure, renal function, and development of antimicrobial resistance in stool flora.

If we critically examine the published studies, some concerns exist: the numbers of children studied are scant; no double-blind, placebo-controlled study has been published, and no children with major urological malformations or renal damage have been included in any study. Thus, we cannot apply these results to children at higher risk, for example, infants with high-grade reflux and renal hypo-dysplasia, or older children with recurrent cystitis and bladder instability. A randomised controlled trial (RCT) evaluating these patients would be very helpful. Another important point is the use of older antibiotics, with an increasing rate of bacterial resistance: co-amoxiclav and co-trimoxazole. The latter, to us surprisingly, is the antibiotic chosen for the prophylaxis in the current RIVUR study. Newer antibiotics, with a better resistance profile, could allow a lower number of recurrences in the prophylaxis arm of studies. No attention has been given to other commonly associated factors, such as constipation and dysfunctional voiding.

From the studies available up to now, we believe it is possible to draw some conclusions, but many uncertainties still remain. Antibiotic prophylaxis is not indicated for children following a first febrile UTI if no or only mild grade I or II reflux is present. Three reasons form the basis of this decision: 1. the meta-analyses of the published data do not show any benefit in this group; 2. there is a relevant risk of inducing bacteria resistance to the antibiotics; 3 . the frequency of reinfection is low (a second episode of febrile UTI occurred in the no-prophylaxis arm of the four studies examined in $3.5 \%, 3 \%$ and $8 \%$ of children with no VUR and grade I and II reflux, respectively). For higher grade reflux, no definite conclusions can be drawn. The reasons for this uncertainty are: 1. the percentage of re-infection is much higher for grade III than for grades 0 , I and II reflux $(28 \%$ of children with grade III reflux of the no-prophylaxis arm); 2. while the meta-analysis demonstrated no significant benefit of antibiotic prophylaxis in this group, the number of treated patients from the published trials was insufficient and will hopefully be addressed by the three as yet unpublished studies.

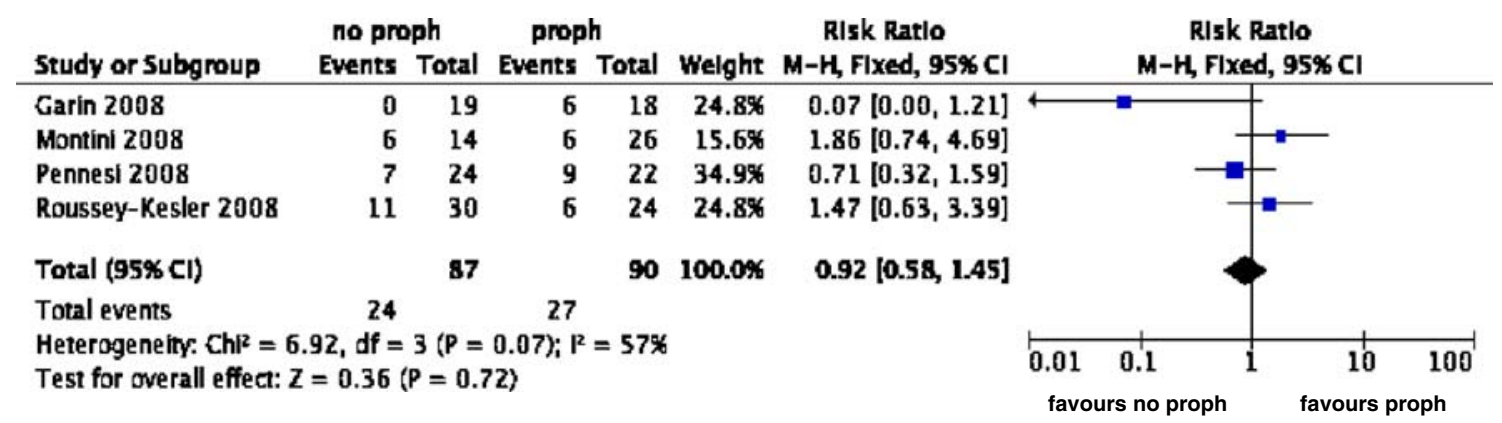

Fig. 2 Meta-analysis of recurrence of febrile UTIs during 1 year of follow-up in children with grade III reflux (proph prophylaxis) 


\section{References}

1. Ferriman A (2007) BMJ readers choose sanitation as greatest medical advance since 1840. BMJ 334:11

2. Arias CA, Murray BE (2009) Antibiotic-resistant bugs in the 21st century-a clinical super-challenge. N Engl J Med 360:439-443

3. Goossens H, Ferech M, Vander Stichele R, Elseviers M, ESAC Project Group (2005) Outpatient antibiotic use in Europe and association with resistance: a cross-national database study. Lancet 365:579-587

4. Chung A, Perera R, Brueggemann AB, Elamin AE, Harnden A, Mayon-White R, Smith S, Crook DW, Mant D (2007) Effect of antibiotic prescribing on antibiotic resistance in individual children in primary care: prospective cohort study. BMJ 335:429

5. Sommet A, Sermet C, Boelle PY, Tafflet M, Bernede C, Guillemot D (2004) No significant decrease in antibiotic use from 1992 to 2000, in the French community. J Antimicrob Chemother 54:524-528

6. Ghiro L, Cracco AT, Sartor M, Comacchio S, Zacchello G, Dall'Amico R (2002) Retrospective study of children with acute pyelonephritis. Nephron 90:8-15

7. Coulthard MG (2009) Vesicoureteric reflux is not a benign condition. Pediatr Nephrol 24:227-232

8. Venhola M, Uhari M (2009) Vesicoureteric reflux, a benign condition. Pediatr Nephrol 24:223-226

9. Beetz R (2006) May we go on with antibacterial prophylaxis for urinary tract infections? Pediatr Nephrol 21:5-13

10. Williams GJ, Wei L, Lee A, Craig JC (2006) Long-term antibiotics for preventing recurrent urinary tract infection in children. Cochrane Database Syst Rev 3:CD001534

11. Garin EH, Olavarria F, Garcia Nieto V, Valenciano B, Campos A, Young L (2006) Clinical significance of primary vesicoureteral reflux and urinary antibiotic prophylaxis after acute pyelonephritis: a multicenter, randomized, controlled study. Pediatrics 117:626-632

12. National Institute for Health and Clinical Excellence. Urinary tract infection in children: diagnosis, treatment and long-term manage- ment. Available at: www.nice.org.uk/cg054. Accessed 30 January 2009

13. Roussey-Kesler G, Gadjos V, Idres N, Horen B, Ichay L, Leclair MD, Raymond F, Grellier A, Hazart I, de Parscau L, Salomon R, Champion G, Leroy V, Guigonis V, Siret D, Palcoux JB, Taque S, Lemoigne A, Nguyen JM, Guyot C (2008) Antibiotic prophylaxis for the prevention of recurrent urinary tract infection in children with low-grade vesicoureteral reflux: results from a prospective randomized study. J Urol 179:674-679

14. Pennesi M, Travan L, Peratoner L, Bordugo A, Cattaneo A, Ronfani L, Minisini S, Ventura A; North East Italy Prophylaxis in VUR study group (2008) Is antibiotic prophylaxis in children with vesicoureteral reflux effective in preventing pyelonephritis and renal scars? A randomized, controlled trial. Pediatrics 121:e1489e1494

15. Montini G, Rigon L, Zucchetta P, Fregonese F, Toffolo A, Gobber D, Cecchin D, Pavanello L, Molinari PP, Maschio F, Zanchetta S, Cassar W, Casadio L, Crivellaro C, Fortunati P, Corsini A, Calderan A, Comacchio S, Tommasi L, Hewitt IK, Da Dalt L, Zacchello G, Dall'Amico R; IRIS Group (2008) Prophylaxis after first febrile urinary tract infection in children? A multicenter, randomized, controlled, noninferiority trial. Pediatrics 122:10641071

16. Brandstrom P, Hansson S (2008) Swedish reflux study in children (abstract). Pediatr Nephrol 23:1703

17. Centre for Kidney Research Children's Hospital at Westmead, Current Research, PRIVENT. Available at http://www.kidneyresearch.org/privent.php (accessed 30 March 2009)

18. Keren R, Carpenter MA, Hoberman A, Shaikh N, Matoo TK, Chesney RW, Matthews R, Gerson AC, Greenfield SP, Fivush B, McLurie GA, Rushton HG, Canning D, Nelson CP, Greenbaum L, Bukowski T, Primack W, Sutherland R, Hosking J, Stewart D, Elder J, Moxey-Mims M, Nyberg L (2008) Rationale and design issues of the Randomized Intervention for Children with Vesicoureteral Reflux (RIVUR) Study. Pediatrics 122(Suppl 5):S240 S250 